\title{
A Berry-Esseen bound for linear combinations of order statistics*
}

\author{
Gyula Pap \\ Institute of Mathematics and Informatics, Lajos Kossuth University \\ Pf.12, H-4010 Debrecen, Hungary \\ Martien C. A. van Zuijlen \\ Department of Mathematics, University of Nijmegen \\ Toernooiveld 1, 6525 ED Nijmegen, The Netherlands
}

Summary. The rate of convergence of the distribution function of a linear combination $n^{-1 / 2} \sum_{j=1}^{n} c_{j n} X_{j: n}$ of order statistics of $n$ independent and identically distributed random variables with a common distribution function $F$ to its normal limit is investigated. Under the assumptions

$$
\begin{aligned}
\left|c_{j n}\right| & =\mathcal{O}\left(n^{-\alpha_{1}}\left(\frac{j}{n}\right)^{-\alpha_{2}}\left(\frac{n-j+1}{n}\right)^{-\alpha_{2}}\right), \\
\left|c_{j n}-c_{j-1, n}\right| & =\mathcal{O}\left(n^{-\beta_{1}}\left(\frac{j}{n}\right)^{-\beta_{2}}\left(\frac{n-j+1}{n}\right)^{-\beta_{2}}\right)
\end{aligned}
$$

with some $\alpha_{1}, \beta_{1} \in \mathbb{R}, \alpha_{2}, \beta_{2} \geq 0$ and

$$
\left|\left(F^{-1}\right)^{\prime}(x)\right|=\mathcal{O}\left(x^{-\kappa}(1-x)^{-\kappa}\right)
$$

with some $0 \leq \kappa<4 / 3$ and appropriate moment conditions a Berry-Esseen bound is given. If the coefficients are generated by a sequence of weight functions of a special structure, then the rate is shown to be $\mathcal{O}\left(n^{-1 / 2}\right)$. Finally, the result is applied for a statistic, which is widely used in auditing.

Key words \& Phrases: linear combinations of order statistics, Berry-Esseen bound, Stringer bound.

${ }^{*}$ Research supported by the Limperg Institute, which is the Interuniversity Research Institute for Accountancy in the Netherlands. 


\section{Motivation, discussion and outline}

Let $X_{1}, X_{2}, \ldots, X_{n}$ be i.i.d. random variables concentrated on $[0,1]$ with a common distribution function $F$. Let $X_{1: n} \leq X_{2: n} \leq \ldots \leq X_{n: n}$ denote the corresponding order statistics. The so-called Stringer bound is defined by

$$
\bar{\mu}_{S T}^{(n)}=p_{0 n}+\sum_{j=1}^{n}\left(p_{n-j+1, n}-p_{n-j, n}\right) X_{j: n},
$$

where $p_{j n}, j=0,1, \ldots, n-1$, is the unique solution of

$$
\sum_{k=0}^{j}\left(\begin{array}{l}
n \\
k
\end{array}\right) p^{k}(1-p)^{n-k}=\alpha
$$

with some fixed $\alpha \in(0,1)$, and $p_{n n}=1$. The Stringer bound is widely used in auditing as a non-parametric $100(1-\alpha) \%$ upper confidence bound for the common mean $\mu=\mathbb{E} X_{1}$. We refer to the papers of Anderson and Teitlebaum (1973), Leslie, Teitlebaum and Anderson (1980), Statistical Models and Analysis in Auditing (1989), Bickel (1992), Pap and Van Zuijlen (1994), (1996) and De Jager, Pap and Van Zuijlen (1997). In Pap and Van Zuijlen (1996) the central limit theorem (CLT)

$$
\frac{\sqrt{n}\left(\bar{\mu}_{S T}^{(n)}-\mu_{n}\right)}{\sigma\left(X_{1}\right)} \stackrel{\mathcal{D}}{\longrightarrow} \mathcal{N}(0,1), \quad \text { as } n \rightarrow \infty
$$

has been shown, where $\stackrel{\mathcal{D}}{\longrightarrow}$ denotes convergence in distribution, $\sigma^{2}\left(X_{1}\right)=\mathbb{E}\left(X_{1}-\mathbb{E} X_{1}\right)^{2}$ denotes the variance of $X_{1}$,

$$
\mu_{n}=\mu+\frac{1}{\sqrt{n}} c(F) z_{1-\alpha}, \quad c(F)=\int_{0}^{1} \sqrt{t(1-t)} d F^{-1}(t),
$$

where $\Phi\left(z_{1-\alpha}\right)=1-\alpha$ with the standard normal distribution function $\Phi$. We remark that

$$
\mathbb{E} \bar{\mu}_{S T}^{(n)}=\mu_{n}+\mathcal{O}\left(n^{-1}\right), \quad \sigma^{2}\left(\bar{\mu}_{S T}^{(n)}\right)=\sigma^{2}\left(X_{1}\right) n^{-1}+o\left(n^{-1}\right)
$$

hence we also have

$$
\frac{\bar{\mu}_{S T}^{(n)}-\mathbb{E} \bar{\mu}_{S T}^{(n)}}{\sigma\left(\bar{\mu}_{S T}^{(n)}\right)} \stackrel{\mathcal{D}}{\longrightarrow} \mathcal{N}(0,1), \quad \text { as } n \rightarrow \infty .
$$

We are interested in the rate of convergence in the above CLT, hence in

$$
\sup _{x}\left|\mathbb{P}\left\{\frac{\bar{\mu}_{S T}^{(n)}-\mathbb{E} \bar{\mu}_{S T}^{(n)}}{\sigma\left(\bar{\mu}_{S T}^{(n)}\right)} \leq x\right\}-\Phi(x)\right| \rightarrow 0, \quad \text { as } n \rightarrow \infty .
$$


Let us consider the following linear combinations of order statistics

$$
L_{n}=n^{-1 / 2} \sum_{j=1}^{n} c_{j n} X_{j: n}
$$

where $c_{j n}=n\left(p_{n-j+1, n}-p_{n-j, n}\right)$. Then $\bar{\mu}_{S T}^{(n)}=p_{0 n}+n^{-1 / 2} L_{n}$, hence

$$
\Delta_{n}=\sup _{x}\left|\mathbb{P}\left\{\frac{L_{n}-\mathbb{E}\left(L_{n}\right)}{\sigma\left(L_{n}\right)} \leq x\right\}-\Phi(x)\right| \rightarrow 0, \quad \text { as } n \rightarrow \infty,
$$

where $\sigma^{2}\left(L_{n}\right)=\sigma^{2}\left(X_{1}\right)+o(1)$ is bounded from below. Corollary 4.2 in Van Zwet (1984) does not provide a Berry-Esseen bound of order $n^{-1 / 2}$, since

Lemma 1. We have

$$
\max _{2 \leq j \leq n}\left|c_{j n}-c_{j-1, n}\right| \geq\left|c_{n n}-c_{n-1, n}\right| \rightarrow c>0, \quad \text { as } n \rightarrow \infty
$$

which implies

$$
n \max _{2 \leq j \leq n}\left|c_{j n}-c_{j-1, n}\right|=n^{2} \max _{0 \leq k \leq n-2}\left|p_{k+2, n}-2 p_{k+1, n}+p_{k n}\right| \rightarrow \infty, \quad \text { as } n \rightarrow \infty .
$$

The proof of this lemma is deferred to the Appendix.

The results of Bjerre (1977) are not applicable since he supposed that $c_{j n}=0$ if $j<\gamma n$ or $j>\delta n$ where $0<\gamma<\delta<1$. The results of Helmers (1981, 1982), Helmers and Hušková $(1984,1986)$ and Serfling (1980) are also not applicable because they assumed that the weights are of the form

$$
c_{j n}=J\left(\frac{j}{n+1}\right) \quad \text { or } \quad c_{j n}=n \int_{(j-1) / n}^{j / n} J(t) d t
$$

with a single weight function $J:(0,1) \rightarrow \mathbb{R}$. One can also consider the weights in (1) in the form

$$
c_{j n}=n \int_{(j-1) / n}^{j / n} J_{n}(t) d t
$$

where $\left\{J_{n}\right\}$ is a sequence of weight functions. Lemma 1 implies that $\lim _{t \rightarrow 0}\left|J_{n}^{\prime}(t)\right|=$ $+\infty$, thus the approach of Section 2.7 in Bentkus, Götze and Van Zwet (1994) also does not provide a Berry-Esseen bound of order $n^{-1 / 2}$. The inconvenient property of the coefficients in the Stringer bound, described in Lemma 1, is connected with the asymptotic behaviour

$$
c_{j n}=n\left(p_{n-j+1, n}-p_{n-j, n}\right)=1+\frac{2 j-n-1}{2 \sqrt{n j(n-j+1)}} z_{1-\alpha}-\frac{1}{n}+\mathcal{O}\left(\frac{n}{j(n-j+1)}\right)^{3 / 2}
$$


(see Pap and Van Zuijlen (1996)), which implies $\sup _{n} \max _{1 \leq j \leq n}\left|c_{j n}\right|<\infty$ and

$$
c_{j n}-c_{j-1, n}=\mathcal{O}\left(\frac{n}{j(n-j+1)}\right)^{3 / 2}
$$

but it does not imply

$$
\sup _{n} n \max _{2 \leq j \leq n}\left|c_{j n}-c_{j-1, n}\right|<\infty .
$$

We remark that Theorem 4.1 of Friedrich (1989) implies

$$
\sup _{x}\left|\mathbb{P}\left\{\frac{L_{n}-\mathbb{E}\left(L_{n}\right)}{\sigma\left(L_{n}\right)} \leq x\right\}-\Phi(x)\right| \leq C_{F} n^{-1 / 2} \quad \text { for all } n \in \mathbb{N}
$$

if $\mathbb{E}\left|X_{1}\right|^{p}<\infty$ for some $p>4$, but the dependence of the constant $C_{F}$ on the underlying distribution is not clarified. In the present paper we will obtain Berry-Esseen bounds for linear combinations of order statistics with coefficients having an asymptotic structure similar to $(2)$.

We start with a generalization of Van Zwet's Berry-Esseen bound (1984, Corollary 4.2). We prove a result where the coefficients of the linear combinations of order statistics are not necessarily generated by weight functions and where the dependence of the bound on the coefficients and on the underlying distribution is also indicated. This theorem provides a bound of order $n^{-1 / 2}$ only if one can guarantee that $\sigma^{2}\left(L_{n}\right)$ (the variance of $L_{n}$ ) is bounded from below. In particular we obtain a Berry-Esseen bound of order $n^{-1 / 2}$ for the Stringer statistic. We also give a statement concerning the convergence of the sequence $\sigma^{2}\left(L_{n}\right)$ in the case where the coefficients are generated by a sequence of weight functions having some special structure (covering the Stringer bound), together with a central limit theorem.

Our starting point is Theorem 1.1 of Van Zwet (1984). We estimate the terms in his Berry-Esseeen bound with the aid of Lemmas 3 and 4, which might be of independent interest.

The paper is organized as follows. Section 2 contains the results. In Section 3 we will give the proof of Theorem 1. In Section 4 we recall a strong law of large numbers due to Van Zwet (1980), and give a corollary of this result, which we will need in Section 5, where we prove the remaining theorems.

\section{Results}

Let $X_{1}, X_{2}, \ldots, X_{n}$ be i.i.d. random variables with a common distribution function $F$. Let $X_{1: n} \leq X_{2: n} \leq \ldots \leq X_{n: n}$ denote the corresponding order statistics. For real numbers 
$c_{1 n}, c_{2 n}, \ldots, c_{n n}, \quad n=1,2, \ldots$, we consider a sequence of normed linear combinations of order statistics

$$
L_{n}=n^{-1 / 2} \sum_{j=1}^{n} c_{j n} X_{j: n}
$$

For $\kappa \geq 0$ let

$$
K_{F}(\kappa):=\sup _{0<x<1} x^{\kappa}(1-x)^{\kappa}\left|\left(F^{-1}\right)^{\prime}(x)\right|,
$$

where $F^{-1}$ is the quantile function of $F$. For convenience we will suppress the dependence on $\kappa$ in the notation $K_{F}(\kappa)$. Let

$$
\begin{array}{r}
\max _{1 \leq j \leq n} n^{\alpha_{1}}\left(\frac{j}{n}\right)^{\alpha_{2}}\left(\frac{n-j+1}{n}\right)^{\alpha_{2}}\left|c_{j n}\right|=a_{n}, \\
\max _{2 \leq j \leq n} n^{\beta_{1}}\left(\frac{j}{n}\right)^{\beta_{2}}\left(\frac{n-j+1}{n}\right)^{\beta_{2}}\left|c_{j n}-c_{j-1, n}\right|=b_{n},
\end{array}
$$

where $\alpha_{1}, \beta_{1} \in \mathbb{R}$ and $\alpha_{2}, \beta_{2} \geq 0$. We note that these values of the parameters are the only cases of interest. (For instance, if $\alpha_{2}<0$, then one can use the result for $\alpha_{2}=0$.) Suppose that

$$
0<\sigma^{2}\left(L_{n}\right)<\infty
$$

and let

$$
\Delta_{n}=\sup _{x}\left|\mathbb{P}\left\{\frac{L_{n}-\mathbb{E} L_{n}}{\sigma\left(L_{n}\right)} \leq x\right\}-\Phi(x)\right| .
$$

Theorem 1. Let $0 \leq \kappa<4 / 3$. Then there exists an absolute constant $C$ and a constant $c=c\left(\alpha_{1}, \alpha_{2}, \beta_{1}, \beta_{2}, \kappa\right)$ (depending on $\alpha_{1}, \alpha_{2}, \beta_{1}, \beta_{2}$ and $\kappa$ but not depending on $n$ or $F$ ) such that

$$
\Delta_{n} \leq C\left(\frac{a_{n}^{3}}{\sigma^{3}\left(L_{n}\right)}\left(A_{n} \mathbb{E}\left|X_{1}\right|^{3}+c \widetilde{A}_{n} K_{F}^{3}\right)+\frac{b_{n}^{2}}{\sigma^{2}\left(L_{n}\right)}\left(B_{n} \mathbb{E} X_{1}^{2}+c \widetilde{B}_{n} K_{F}^{2}\right)\right) n^{-1 / 2}
$$

where

$$
\begin{gathered}
A_{n}:= \begin{cases}n^{-3\left(\alpha_{1}-\alpha_{2}\right)} & \text { if } \alpha_{1} \geq \alpha_{2}, \\
0 & \text { if } \alpha_{1}<\alpha_{2},\end{cases} \\
\widetilde{A}_{n}:= \begin{cases}n^{-3 \alpha_{1}} & \text { if } \alpha_{1}<\alpha_{2}, \alpha_{2}+\kappa<\frac{4}{3}, \\
n^{-3 \alpha_{1}} \log ^{3} n & \text { if } \alpha_{1}<\alpha_{2}, \alpha_{2}+\kappa=\frac{4}{3}, \\
n^{-3\left(\alpha_{1}-\alpha_{2}-\kappa\right)-4} & \text { if } \alpha_{1}<\alpha_{2}, \alpha_{2}+\kappa>\frac{4}{3}, \\
0 & \text { if } \alpha_{1} \geq \alpha_{2},\end{cases} \\
B_{n}:= \begin{cases}n^{-2\left(\beta_{1}-\beta_{2}-1\right)} & \text { if } \beta_{1} \geq \beta_{2}+1, \\
0 & \text { if } \beta_{1}<\beta_{2}+1,\end{cases}
\end{gathered}
$$




$$
\widetilde{B}_{n}:= \begin{cases}n^{-2\left(\beta_{1}-1\right)} & \text { if } \beta_{1}<\beta_{2}+1, \quad \beta_{2}+\kappa<\frac{5}{2}, \\ n^{-2\left(\beta_{1}-1\right)} \log n & \text { if } \beta_{1}<\beta_{2}+1, \quad \beta_{2}+\kappa=\frac{5}{2} \\ n^{-2\left(\beta_{1}-\beta_{2}-\kappa\right)-3} & \text { if } \beta_{1}<\beta_{2}+1, \quad \beta_{2}+\kappa>\frac{5}{2} \\ 0 & \text { if } \beta_{1} \geq \beta_{2}+1 .\end{cases}
$$

We note that Theorem 1 provides a Berry-Esseen bound of order $n^{-1 / 2}$ if $\sigma^{2}\left(L_{n}\right)$ is bounded from below, $K_{F}<\infty, a_{n}$ and $b_{n}$ are bounded from above and

$$
\alpha_{1}>\left(\alpha_{2}+\kappa-\frac{4}{3}\right)^{+}, \quad \beta_{1}>\left(\beta_{2}+\kappa-\frac{5}{2}\right)^{+}+1
$$

(If $\alpha_{2}+\kappa \neq 4 / 3$ then $\alpha_{1}=\left(\alpha_{2}+\kappa-4 / 3\right)^{+}$is also allowed, and a similar remark can be formulated for $\beta_{1}$.)

Friedrich $(1985,1989)$ imposed an extra condition of the type

$$
\left|c_{j n}-2 c_{j-1, n}+c_{j-2, n}\right|=\mathcal{O}\left(n^{-\gamma_{1}}\left(\frac{j}{n}\right)^{-\gamma_{2}}\left(\frac{n-j+1}{n}\right)^{-\gamma_{2}}\right) .
$$

In Theorem 4.1 in Friedrich (1989) the special case $\alpha_{1}=0, \quad \beta_{1}=1, \gamma_{1}=2$ and $\delta:=\alpha_{2}=\beta_{2}-1=\gamma_{2}-2 \in(0,1 / 4)$ has been considered. Under the moment conditions $\mathbb{E}\left|X_{1}\right|^{p}<\infty$ with some $p>4 /(1-4 \delta)$ and $\sigma^{2}\left(L_{n}\right)>0$ he proved a Berry-Esseen bound $\Delta_{n}=\mathcal{O}\left(n^{-1 / 2}\right)$ without specifying the dependence of the constant (hidden in $\left.\mathcal{O}\left(n^{-1 / 2}\right)\right)$ on the parameter $\delta$ and on the underlying distribution.

The function

$$
\frac{1}{\left(F^{-1}\right)^{\prime}(x)}=F^{\prime}\left(F^{-1}(x)\right)
$$

is called the density quantile function of the distribution $F$, hence $K_{F}(\kappa)<\infty$ is related with the tail behaviour of the distribution $F$ (cf. Parzen (1979)) . If the left and right tail exponent of the distribution $F$ are $\gamma_{1}$ and $\gamma_{2}$, respectively, then

$$
\left(F^{-1}\right)^{\prime}(x)=L(x) x^{-\gamma_{1}}(1-x)^{-\gamma_{2}},
$$

where $L:(0,1) \rightarrow \mathbb{R}$ is slowly varying from the right at $x=0$ and from the left at $x=1$. We remark that $K_{F}(0)<\infty$ in the case of the uniform distribution, and $K_{F}(1)<\infty$ in the case of the normal, exponential, Weibull, extreme value and logistic distributions. Note also that in the case of the lognormal distribution we have $K_{F}(\kappa)<\infty$ only if $\kappa>1$. In the case of the Cauchy distribution $K_{F}(\kappa)<\infty$ if $\kappa \geq 2$.

We remark the connection with the refined Parzen classification of distribution functions (see Schuster (1984)): if $K_{F}(\kappa)<\infty$ with some $\kappa<1$ then the distribution has 
short or medium-short tails; if $K_{F}(1)<\infty$ then the distribution has medium-medium tails; if one of the tails of the distribution is medium-long or long then $K_{F}(1)=\infty$. (The connection with the extreme value index of the distribution - classifying the types of distributions leading to the three possible limiting distributions of extremes — is not clear yet.)

We remark also that if $K_{F}(\kappa)<\infty$ for some $\kappa \in[0,1]$ then the existence of all moments of the underlying distribution is guaranteed. If $K_{F}(\kappa)<\infty$ for some $\kappa>1$ then $\mathbb{E}\left|X_{1}\right|^{p}<\infty$ for $p<1 /(\kappa-1)$.

We can apply Theorem 1 for the Stringer bound $\bar{\mu}_{S T}^{(n)}$ defined in Section 1:

Corollary 1. Let $0 \leq \kappa<4 / 3$. Then there exists an absolute constant $C$ and a constant $c=c(\kappa)$ (depending on $\kappa$ but not depending on $n$ or $F$ ) such that for the Stringer bound we have

$$
\Delta_{n} \leq C\left(\frac{\mathbb{E}\left|X_{1}\right|^{3}}{\sigma^{3}\left(L_{n}\right)}+\frac{\left.c(\kappa) K_{F}^{2}(\kappa) \widetilde{B}_{n}\right)}{\sigma^{2}\left(L_{n}\right)}\right) n^{-1 / 2},
$$

where

$$
\widetilde{B}_{n}= \begin{cases}n^{-1} & \text { if } \kappa<1, \\ n^{-1} \log n & \text { if } \kappa=1, \\ n^{2 \kappa-3} & \text { if } 1<\kappa<4 / 3 .\end{cases}
$$

If $K_{F}(\kappa)<\infty$ then the leading term of the bound is the first term (which has the classical form) since the second term in the parentheses is of order $O\left(n^{-1 / 3}\right)$ for all $0 \leq \kappa<4 / 3$.

Next we prove a CLT together with convergence of $\sigma^{2}\left(L_{n}\right)$. Consider sequences of linear combinations of order statistics of the following form:

$$
L_{n}=n^{-1 / 2} \sum_{j=1}^{n} c_{j n} X_{j: n}, \quad \widehat{L}_{n}=n^{-1 / 2} \sum_{j=1}^{n} \widehat{c}_{j n} X_{j: n},
$$

where

$$
c_{j n}=n \int_{(j-1) / n}^{j / n} J_{n}(t) d t, \quad \widehat{c}_{j n}=J_{n}\left(\frac{j}{n+1}\right)
$$

and the weight functions $J_{n}:(0,1) \rightarrow \mathbb{R}, n=1,2, \ldots$, have the form:

$$
J_{n}(t)=\sum_{i=0}^{k} \varphi_{i}(t) n^{-\delta_{i}}, \quad \varphi_{i}(t)=t^{-\gamma_{i}}(1-t)^{-\gamma_{i}} \psi_{i}(t)
$$

with some functions $\psi_{i}:(0,1) \rightarrow \mathbb{R}, i=0,1, \ldots, k$, and with $0=\delta_{0}<\delta_{1}<\cdots<\delta_{k}$.

Theorem 2. Suppose that 
(i) $\delta_{0}=0,1 / 2 \leq \delta_{1}<\delta_{2}<\cdots<\delta_{k}$, and $0 \leq \gamma_{i}<\delta_{i}+1 / 2, \quad i=0,1, \ldots, k$,

(ii) we have

$$
\left|\psi_{0}(s)-\psi_{0}(t)\right| \leq \Lambda_{0}|s-t|^{\lambda_{0}}
$$

for all $0<s<t<1$ and for some $\lambda_{0}>1 / 2$ and $\Lambda_{0} \geq 0$,

(iii) $\psi_{1}, \ldots, \psi_{k}$ are bounded and Lebesgue measurable,

(iv) $\mathbb{E}\left|X_{1}\right|^{m}<\infty$ for some

$$
m>\max _{0 \leq i \leq k}\left(\frac{1}{2}-\gamma_{i}+\delta_{i}\right)^{-1}
$$

Then

$$
L_{n}-\mathbb{E} L_{n} \stackrel{\mathcal{D}}{\longrightarrow} \mathcal{N}\left(0, \sigma^{2}\left(\varphi_{0}, F\right)\right)
$$

and

$$
\lim _{n \rightarrow \infty} \sigma^{2}\left(L_{n}\right)=\sigma^{2}\left(\varphi_{0}, F\right)
$$

where

$$
\sigma^{2}\left(\varphi_{0}, F\right)=\int_{0}^{1} \int_{0}^{1} \varphi_{0}(s) \varphi_{0}(t)(s \wedge t-s t) d F^{-1}(s) d F^{-1}(t) .
$$

Moreover, under the extra condition

(v) we have

$$
\left|\psi_{1}(s)-\psi_{1}(t)\right| \leq \Lambda_{1}|s-t|^{\lambda_{1}}
$$

for all $0<s<t<1$ and for some $\lambda_{1}>0$ and $\Lambda_{1} \geq 0$

we also have

$$
\widehat{L}_{n}-\mathbb{E} \widehat{L}_{n} \stackrel{\mathcal{D}}{\longrightarrow} \mathcal{N}\left(0, \sigma^{2}\left(\varphi_{0}, F\right)\right)
$$

and

$$
\lim _{n \rightarrow \infty} \sigma^{2}\left(\widehat{L}_{n}\right)=\sigma^{2}\left(\varphi_{0}, F\right) .
$$

We mention that central limit theorems in the case of sequences of weight functions can also be found in Shorack and Wellner (1986, Theorem 1 and 6 in Chapter 19). However, these results cannot be applied in general in the situation considered in Theorem 2. Furthermore we note that in the special case of Theorem 2 where $k=0$ we have a single weight function and our Theorem 2 is slightly weaker than Theorem 1 in Mason (1981).

Next we will prove a Berry-Esseen bound for $L_{n}$. Clearly a similar result holds for $\widehat{L}_{n}$. 
For $i=0,1, \ldots, k$ let

$$
\sup _{0<x<y<1}|x-y|^{-\lambda_{i}}\left|\psi_{i}(x)-\psi_{i}(y)\right|=\Lambda_{i}
$$

with some $0 \leq \lambda_{i} \leq 1$.

Theorem 3. Let $0 \leq \kappa<4 / 3$ and $0 \leq \gamma_{i}<1, i=1, \ldots, k$. Then there exists an absolute constant $C$ and a constant $c=c\left(\kappa, \lambda_{i}, \delta_{i}, \gamma_{i} ; i \in\{0,1, \ldots, k\}\right)$ (depending on $\kappa, \lambda_{i}, \delta_{i}, \gamma_{i} ; i \in\{0,1, \ldots, k\}$, but not depending on $n$ or $F$ ) such that

$$
\begin{gathered}
\Delta_{n} \leq \frac{C}{n^{1 / 2}}\left(\frac{1}{\sigma^{3}\left(L_{n}\right)} \sum_{i=0}^{k} \frac{\left\|\psi_{i}\right\|_{\infty}^{3}}{\left(1-\gamma_{i}\right)^{3}}\left(A_{i n} \mathbb{E}\left|X_{1}\right|^{3}+c \widetilde{A}_{i n} K_{F}^{3}\right)+\right. \\
+\frac{1}{\sigma^{2}\left(L_{n}\right)} \sum_{i=0}^{k}\left\{\Lambda_{i}^{2}\left(B_{i n} \mathbb{E} X_{1}^{2}+c \widetilde{B}_{i n} K_{F}^{2}\right)+\right. \\
\left.\left.\quad+\frac{\left\|\psi_{i}\right\|_{\infty}^{2}}{\left(1-\gamma_{i}\right)^{2}}\left(D_{i n} \mathbb{E} X_{1}^{2}+c \widetilde{D}_{i n} K_{F}^{2}\right)\right\}\right),
\end{gathered}
$$

where

$$
\begin{aligned}
& A_{\text {in }}:= \begin{cases}n^{-3\left(\delta_{i}-\gamma_{i}\right)} & \text { if } \delta_{i} \geq \gamma_{i}, \\
0 & \text { if } \delta_{i}<\gamma_{i},\end{cases} \\
& \widetilde{A}_{\text {in }}:= \begin{cases}n^{-3 \delta_{i}} & \text { if } \delta_{i}<\gamma_{i}, \gamma_{i}+\kappa<\frac{4}{3}, \\
n^{-3 \delta_{i}} \log ^{3} n & \text { if } \delta_{i}<\gamma_{i}, \gamma_{i}+\kappa=\frac{4}{3}, \\
n^{-3\left(\delta_{i}-\gamma_{i}-\kappa\right)-4} & \text { if } \delta_{i}<\gamma_{i}, \gamma_{i}+\kappa>\frac{4}{3}, \\
0 & \text { if } \delta_{i} \geq \gamma_{i},\end{cases} \\
& B_{\text {in }}:= \begin{cases}n^{-2\left(\delta_{i}+\lambda_{i}-\gamma_{i}-1\right)} & \text { if } \delta_{i}+\lambda_{i} \geq \gamma_{i}+1, \\
0 & \text { if } \delta_{i}+\lambda_{i}<\gamma_{i}+1\end{cases} \\
& \widetilde{B}_{i n}:=\left\{\begin{array}{lll}
n^{-2\left(\delta_{i}+\lambda_{i}-1\right)} & \text { if } \delta_{i}+\lambda_{i}<\gamma_{i}+1, \gamma_{i}+\kappa<\frac{5}{2}, \\
n^{-2\left(\delta_{i}+\lambda_{i}-1\right)} \log n & \text { if } \delta_{i}+\lambda_{i}<\gamma_{i}+1, \gamma_{i}+\kappa=\frac{5}{2}, \\
n^{-2\left(\delta_{i}+\lambda_{i}-\gamma_{i}-\kappa\right)-1} & \text { if } \delta_{i}+\lambda_{i}<\gamma_{i}+1, \gamma_{i}+\kappa>\frac{5}{2}, \\
0 & \text { if } \delta_{i}+\lambda_{i} \geq \gamma_{i}+1,
\end{array}\right. \\
& D_{\text {in }}:= \begin{cases}n^{-2\left(\delta_{i}-\gamma_{i}-1\right)} & \text { if } \delta_{i} \geq \gamma_{i}+1, \\
0 & \text { if } \delta_{i}<\gamma_{i}+1,\end{cases} \\
& \widetilde{D}_{i n}:= \begin{cases}n^{-2 \delta_{i}} & \text { if } \delta_{i}<\gamma_{i}+1, \quad \gamma_{i}+\kappa<\frac{3}{2}, \\
n^{-2 \delta_{i}} \log n & \text { if } \delta_{i}<\gamma_{i}+1, \quad \gamma_{i}+\kappa=\frac{3}{2}, \\
n^{-2\left(\delta_{i}-\gamma_{i}-\kappa\right)-3} & \text { if } \delta_{i}<\gamma_{i}+1, \quad \gamma_{i}+\kappa>\frac{3}{2}, \\
0 & \text { if } \delta_{i} \geq \gamma_{i}+1 .\end{cases}
\end{aligned}
$$


We note that Theorem 3 provides a Berry-Esseen bound of order $n^{-1 / 2}$ if the conditions of Theorem 2 hold, $\sigma^{2}\left(\varphi_{0}, F\right)>0, K_{F}, \Lambda_{0}, \Lambda_{1}, \ldots, \Lambda_{k}<\infty$, and for $i=0,1, \ldots, k$

$$
\delta_{i}>\max \left\{\left(\gamma_{i}+\kappa-\frac{4}{3}\right)^{+},\left(\gamma_{i}+\kappa-\frac{5}{2}\right)^{+}+1-\lambda_{i}\right\} .
$$

(In some cases equality is also allowed; see the note after Theorem 1.) From Theorem 3 one might have the feeling that the speed of convergence can be better than $n^{-1 / 2}$. However for $i=0$ we have necessarily $\delta_{0}=0$ and $0 \leq \gamma_{0}<1 / 2$. Hence if $\gamma_{0}=0$ then in the case $\lambda_{0}=1$ we have $B_{0 n}=1$, and in the case $\lambda_{0}<1$ we have $\widetilde{B}_{0 n}=n^{2\left(1-\lambda_{0}\right)} \geq 1$. If $0<\gamma_{0}<1 / 2$ then the inequalities $\lambda_{0}<\gamma_{0}+1$ and $\gamma_{0}+\kappa<5 / 2$ imply $\widetilde{B}_{0 n}=n^{2\left(1-\lambda_{0}\right)} \geq 1$. Consequently, indeed, the speed of convergence $n^{-1 / 2}$ cannot be improved.

Helmers $(1981,1982)$ covered the case where $k=0, \gamma_{0}=0$ and $\lambda_{0}=1$. Helmers and Hušková $(1984,1986)$ have considered essentially the case where $k=0,0 \leq \kappa<5 / 4$ and $\left|\phi_{0}^{\prime \prime}(t)\right| \leq c t^{-2}(1-t)^{-2}$ for $0<t<1$.

\section{The proof of Theorem 1}

For the proof of Theorem 1 we need some preparations.

Lemma 2. Let $y \in \mathbb{R}$. Then there exist constants $c_{1}(y)>0$ and $c_{2}(y)>0$ (depending only on $y$ ) such that for all $k \in \mathbb{N}$ with $k>-y$ we have

$$
c_{1}(y) k^{y} \leq \frac{\Gamma(k+y)}{\Gamma(k)} \leq c_{2}(y) k^{y}
$$

Proof. By Stirling's formula we have for fixed $a \in \mathbb{R}$

$$
\log \Gamma(x+a)=\left(x+a-\frac{1}{2}\right) \log x-x+\frac{1}{2} \log 2 \pi+\mathcal{O}\left(x^{-1}\right), \quad \text { as } \quad x \rightarrow \infty,
$$

and consequently, for fixed $y \in \mathbb{R}$,

$$
\log \Gamma(k+y)-\log \Gamma(k)-y \log k=\mathcal{O}\left(k^{-1}\right), \quad \text { as } \quad k \rightarrow \infty .
$$

Hence

$$
\lim _{k \rightarrow \infty}(\log \Gamma(k+y)-\log \Gamma(k)-y \log k)=0
$$


which implies

$$
\lim _{k \rightarrow \infty} k^{-y} \frac{\Gamma(k+y)}{\Gamma(k)}=1
$$

from which the assertion follows.

Let $U_{1}, U_{2}, \ldots, U_{n}$ be i.i.d. random variables with a common uniform distribution on $(0,1)$. We take $U_{0: n}=0, U_{n+1: n}=1$. For $r, s>0$ let $b_{r, s}$ be the beta density

$$
b_{r, s}(x)=\frac{x^{r-1}(1-x)^{s-1}}{b(r, s)}, \quad 0<x<1,
$$

where

$$
b(r, s)=\int_{0}^{1} x^{r-1}(1-x)^{s-1} d x=\frac{\Gamma(r) \Gamma(s)}{\Gamma(r+s)}
$$

is the beta function.

The following lemmas will be used intensively and might be of independent interest.

Lemma 3. For every $\gamma, \delta \in \mathbb{R}$ there exist constants $c_{1}(\gamma, \delta)>0$ and $c_{2}(\gamma, \delta)>0$ (depending only on $\gamma$ and $\delta$ ) such that for all $n \in \mathbb{N}$ and $j \in\{1, \ldots, n\}$ with $-\gamma<j<n+\delta+1$ we have

$$
c_{1}(\gamma, \delta)\left(\frac{j}{n}\right)^{\gamma}\left(\frac{n-j+1}{n}\right)^{\delta} \leq \mathbb{E} U_{j: n}^{\gamma}\left(1-U_{j: n}\right)^{\delta} \leq c_{2}(\gamma, \delta)\left(\frac{j}{n}\right)^{\gamma}\left(\frac{n-j+1}{n}\right)^{\delta}
$$

Proof. We have

$$
\begin{aligned}
\mathbb{E} U_{j: n}^{\gamma}\left(1-U_{j: n}\right)^{\delta} & =\int_{0}^{1} x^{\gamma}(1-x)^{\delta} b_{j, n-j+1}(x) d x= \\
& =\frac{\Gamma(n+1)}{\Gamma(j) \Gamma(n-j+1)} \cdot \frac{\Gamma(j+\gamma) \Gamma(n-j+1+\delta)}{\Gamma(n+1+\gamma+\delta)}
\end{aligned}
$$

hence by Lemma 2 the proof can be completed.

Lemma 4. For every $\gamma, \delta \in \mathbb{R}$ there exists a constant $c(\gamma, \delta)>0$ (depending only on $\gamma$ and $\delta$ ) such that for all $n \in \mathbb{N}$ and

(i) $j \in\{2, \ldots, n\}$ with $-2 \gamma-1<j<n+2 \delta+3$,

(ii) $j=1$ with $-2 \gamma-1<j<n+2 \delta+1$,

(iii) $j=n+1$ with $-2 \gamma+1<j<n+2 \delta+3$ 
we have

$$
\sqrt{\mathbb{E}\left(\int_{U_{j-1: n}}^{U_{j: n}} x^{\gamma}(1-x)^{\delta} d x\right)^{2}} \leq c(\gamma, \delta) \frac{1}{n}\left(\frac{j}{n}\right)^{\gamma}\left(\frac{n-j+2}{n}\right)^{\delta}
$$

Proof. First let $j \in\{2, \ldots, n\}$ and $\gamma>0, \delta>0$ with $-2 \gamma+1<j<n+2 \delta+1$. Then the function $x \mapsto x^{\gamma}(1-x)^{\delta}$ is monotone increasing on $\left[0, x_{0}\right]$ and monotone decreasing on $\left[x_{0}, 1\right]$, where $x_{0}=\gamma /(\gamma+\delta)$. For some $c_{\gamma \delta}>0$ we have

$$
\begin{aligned}
& \mathbb{E}\left(\int_{U_{j-1: n}}^{U_{j: n}} x^{\gamma}(1-x)^{\delta} d x\right)^{2} \leq \\
& \quad \leq \mathbb{E}\left(U_{j: n}-U_{j-1: n}\right)^{2}\left(U_{j-1: n}^{2 \gamma}\left(1-U_{j-1: n}\right)^{2 \delta}+U_{j: n}^{2 \gamma}\left(1-U_{j: n}\right)^{2 \delta}\right)+ \\
& \quad+c_{\gamma \delta} \mathbb{E}\left\{\left(U_{j: n}-U_{j-1: n}\right)^{2} \mid U_{j-1: n}<x_{0}<U_{j: n}\right\} \mathbb{P}\left\{U_{j-1: n}<x_{0}<U_{j: n}\right\} .
\end{aligned}
$$

Using that $U_{j-1: n} / U_{j: n}$ and $U_{j: n}$ are independent random variables and $U_{j-1: n} / U_{j: n} \stackrel{\mathcal{D}}{=}$ $U_{j-1: j-1}$ (see Corollary 1.6.3 in Reiss (1989)), and applying Lemma 3 we obtain

$$
\begin{aligned}
& \mathbb{E}\left(U_{j: n}-U_{j-1: n}\right)^{2} U_{j: n}^{2 \gamma}\left(1-U_{j: n}\right)^{2 \delta}=\mathbb{E}\left(1-\frac{U_{j-1: n}}{U_{j: n}}\right)^{2} U_{j: n}^{2 \gamma+2}\left(1-U_{j: n}\right)^{2 \delta}= \\
& \quad=\mathbb{E}\left(1-U_{j-1: j-1}\right)^{2} \mathbb{E} U_{j: n}^{2 \gamma+2}\left(1-U_{j: n}\right)^{2 \delta}=\mathcal{O}\left(\frac{1}{(j-1)^{2}} \frac{j^{2 \gamma+2}(n-j+1)^{2 \delta}}{n^{2 \gamma+2 \delta+2}}\right)= \\
& \quad=\mathcal{O}\left(\frac{j^{2 \gamma}(n-j+2)^{2 \delta}}{n^{2 \gamma+2 \delta+2}}\right) .
\end{aligned}
$$

In a similar way using that $\left(1-U_{j: n}\right) /\left(1-U_{j-1: n}\right)$ and $1-U_{j-1: n}$ are independent random variables and $\left(1-U_{j: n}\right) /\left(1-U_{j-1: n}\right) \stackrel{\mathcal{D}}{=} 1-U_{n-j+1: n-j+1} \quad$ (see Corollary 1.6.2 in Reiss (1989)), and applying again Lemma 3 we obtain

$$
\begin{gathered}
\mathbb{E}\left(U_{j: n}-U_{j-1: n}\right)^{2} U_{j-1: n}^{2 \gamma}\left(1-U_{j-1: n}\right)^{2 \delta}=\mathbb{E}\left(\frac{U_{j: n}-U_{j-1: n}}{1-U_{j-1: n}}\right)^{2} U_{j-1: n}^{2 \gamma}\left(1-U_{j-1: n}\right)^{2 \delta+2}= \\
=\mathbb{E} U_{n-j+1: n-j+1}^{2} \mathbb{E} U_{j-1: n}^{2 \gamma}\left(1-U_{j-1: n}\right)^{2 \delta+2}=\mathcal{O}\left(\frac{j^{2 \gamma}(n-j+2)^{2 \delta}}{n^{2 \gamma+2 \delta+2}}\right) .
\end{gathered}
$$

For $0<u<x_{0}<v<1$ we have

$$
\mathbb{P}\left\{U_{j-1: n}<u, U_{j: n}>v \mid U_{j-1: n}<x_{0}<U_{j: n}\right\}=\frac{u^{j-1}(1-v)^{n-j+1}}{x_{0}^{j-1}\left(1-x_{0}\right)^{n-j+1}},
$$

consequently the conditional density of $\left(U_{j-1: n}, U_{j: n}\right)$ with respect to the condition $\left\{U_{j-1: n}<x_{0}<U_{j: n}\right\}$ has the form

$$
(u, v) \mapsto \begin{cases}(j-1)(n-j+1) \frac{u^{j-2}(1-v)^{n-j}}{x_{0}^{j-1}\left(1-x_{0}\right)^{n-j+1}} & \text { for } 0<u<x_{0}<v<1 \\ 0 & \text { otherwise. }\end{cases}
$$


Berry-Esseen bound for L-statistics

Using this conditional density, straightforward but somewhat tedious computations show that

$$
\mathbb{E}\left\{\left(U_{j: n}-U_{j-1: n}\right)^{2} \mid U_{j-1: n}<x_{0}<U_{j: n}\right\}=\mathcal{O}\left(\frac{n^{2}}{j^{2}(n-j+1)^{2}}\right) .
$$

Moreover,

$$
\begin{aligned}
& \mathbb{P}\left\{U_{j-1: n}<x_{0}<U_{j: n}\right\}=\left(\begin{array}{c}
n \\
j-1
\end{array}\right) x_{0}^{j-1}\left(1-x_{0}\right)^{n-j+1}= \\
& =x_{0}^{-1}\left(1-x_{0}\right)^{-1} \frac{j(n-j+2)}{(n+1)(n+2)}\left(\begin{array}{c}
n+2 \\
j
\end{array}\right) x_{0}^{j}\left(1-x_{0}\right)^{n-j+2} \leq \\
& \leq x_{0}^{-1}\left(1-x_{0}\right)^{-1} \frac{j(n-j+2)}{(n+1)(n+2)}=\mathcal{O}\left(\frac{j(n-j+1)}{n^{2}}\right) .
\end{aligned}
$$

In a similar way one can show for arbitrary $k \in \mathbb{N}$

$$
\mathbb{P}\left\{U_{j-1: n}<x_{0}<U_{j: n}\right\}=\mathcal{O}\left(\frac{j^{k}(n-j+1)^{k}}{n^{2 k}}\right) .
$$

Collecting the estimates from (7), (8), (9), (10) and (11) we conclude the statement for $j \in\{2, \ldots, n\}, \gamma>0, \delta>0$ and $-2 \gamma+1<j<n+2 \delta+1$. In the cases where $\gamma \leq 0$ or $\delta \leq 0$ the proof can be carried out in the same way.

If $j \in\{2, \ldots, n\}, \gamma \neq-1, \delta \leq 0$ and $-2 \gamma-1<j \leq-2 \gamma+1, j<n+2 \delta+1$ then in an analogous way we obtain

$$
\begin{aligned}
& \mathbb{E}\left(\int_{U_{j-1: n}}^{U_{j: n}} x^{\gamma}(1-x)^{\delta} d x\right)^{2} \leq(\gamma+1)^{-2} \mathbb{E}\left(U_{j: n}^{\gamma+1}-U_{j-1: n}^{\gamma+1}\right)^{2}\left(1-U_{j: n}\right)^{2 \delta}= \\
& \quad=(\gamma+1)^{-2} \mathbb{E}\left(1-\frac{U_{j-1: n}^{\gamma+1}}{U_{j: n}^{\gamma+1}}\right)^{2} \mathbb{E} U_{j: n}^{2 \gamma+2}\left(1-U_{j: n}\right)^{2 \delta}=\mathcal{O}\left(\frac{j^{2 \gamma}(n-j+1)^{2 \delta}}{n^{2 \gamma+2 \delta+2}}\right),
\end{aligned}
$$

since

$$
\mathbb{E}\left(1-\frac{U_{j-1: n}^{\gamma+1}}{U_{j: n}^{\gamma+1}}\right)^{2}=\mathbb{E}\left(1-U_{j-1: j-1}^{\gamma+1}\right)^{2}=\frac{2(\gamma+1)^{2}}{(j+\gamma)(j+2 \gamma+1)}
$$

If $j \in\{2, \ldots, n\}, \gamma=-1, \delta \leq 0$ and $-2 \gamma-1<j \leq-2 \gamma+1, j<n+2 \delta+1$ then

$$
\begin{gathered}
\mathbb{E}\left(\int_{U_{j-1: n}}^{U_{j: n}} x^{-1}(1-x)^{\delta} d x\right)^{2} \leq \mathbb{E}\left(\log U_{j: n}-\log U_{j-1: n}\right)^{2}\left(1-U_{j: n}\right)^{2 \delta}= \\
\quad=\mathbb{E}\left(\log \frac{U_{j-1: n}}{U_{j: n}}\right)^{2} \mathbb{E}\left(1-U_{j: n}\right)^{2 \delta}=\mathcal{O}\left(\frac{j^{-2}(n-j+1)^{2 \delta}}{n^{2 \delta}}\right)
\end{gathered}
$$

since

$$
\mathbb{E}\left(\log \frac{U_{j-1: n}}{U_{j: n}}\right)^{2}=\mathbb{E}\left(\log U_{j-1: j-1}\right)^{2}=\frac{2}{(j-1)^{2}}
$$


Since the case $\delta>0$ is similar, we conclude the statement for $j \in\{2, \ldots, n\},-2 \gamma-1<$ $j \leq-2 \gamma+1$ and $j<n+2 \delta+1$.

If $j \in\{2, \ldots, n\}$ and $n+2 \delta+1 \leq j<n+2 \delta+3, j>-2 \gamma+1$ then again similar arguments can be used.

Let now $j=1$ with $-2 \gamma-1<j<n+2 \delta+1$. The condition $j>-2 \gamma-1$ implies $\gamma>-1$. If $\delta \leq 0$ then by Lemma 3

$$
\begin{aligned}
& \mathbb{E}\left(\int_{0}^{U_{1: n}} x^{\gamma}(1-x)^{\delta} d x\right)^{2} \leq \mathbb{E}\left(\left(1-U_{1: n}\right)^{\delta} \int_{0}^{U_{1: n}} x^{\gamma} d x\right)^{2}= \\
& =(\gamma+1)^{-2} \mathbb{E} U_{1: n}^{2 \gamma+2}\left(1-U_{1: n}\right)^{2 \delta}=\mathcal{O}\left(n^{-2 \gamma-2}\right) .
\end{aligned}
$$

If $\delta>0$ then

$$
\mathbb{E}\left(\int_{0}^{U_{1: n}} x^{\gamma}(1-x)^{\delta} d x\right)^{2} \leq \mathbb{E}\left(\int_{0}^{U_{1: n}} x^{\gamma} d x\right)^{2}=(\gamma+1)^{-2} \mathbb{E} U_{1: n}^{2 \gamma+2}=\mathcal{O}\left(n^{-2 \gamma-2}\right)
$$

The case $j=n+1$ can be handled as the case $j=1$, so that the proof is completed.

Proof of Theorem 1. We shall find it convenient to introduce i.i.d. random variables $U_{1}, U_{2}, \ldots, U_{n}$ with a common uniform distribution on $(0,1)$ and pretend that $X_{j}=$ $F^{-1}\left(U_{j}\right)$ for $j=1, \ldots, n$. We take $X_{0: n}=-\infty, X_{n+1: n}=+\infty$. The rank of $U_{j}$ among $U_{1}, \ldots, U_{n}$ will be denoted by $R_{j}$, and we define

$$
K_{1}=\min \left\{R_{n-1}, R_{n}\right\}, \quad K_{2}=\max \left\{R_{n-1}, R_{n}\right\}
$$

Furthermore we define the functions $H_{1 j}, H_{2 j}$ for $j=1, \ldots, n$ and $M_{0}, M_{1}, M_{2}$ by

$$
\begin{gathered}
H_{1 j}(x)=\int_{0}^{x} y b_{j, n-j+1}(y) d F^{-1}(y), \quad H_{2 j}(x)=\int_{x}^{1}(1-y) b_{j, n-j+1}(y) d F^{-1}(y), \\
M_{0}(x)=\int_{-\infty}^{x} F^{2}(y) d y, \quad M_{1}(x)=\int_{-\infty}^{x} F(y)(1-F(y)) d y, \quad M_{2}(x)=\int_{x}^{\infty}(1-F(y))^{2} d y .
\end{gathered}
$$

From Theorem 1.1 of Van Zwet (1984) we have

$$
\Delta_{n} \leq C\left(\frac{\mathbb{E}|W|^{3}}{\sigma^{3}\left(L_{n}\right)}+\frac{\mathbb{E} Z^{2}}{\sigma^{2}\left(L_{n}\right)}\right) n^{-1 / 2}
$$

where

$$
\begin{aligned}
W & =\frac{1}{n} \sum_{j=1}^{n} c_{j n}\left(H_{1 j}\left(U_{1}\right)-H_{2 j}\left(U_{1}\right)\right) \\
Z & =n \sum_{j=1}^{K_{1}}\left(c_{j+1, n}-c_{j n}\right)\left(M_{0}\left(X_{j: n}\right)-M_{0}\left(X_{j-1: n}\right)\right)-
\end{aligned}
$$


Berry-Esseen bound for L-statistics

$$
\begin{aligned}
& \quad-n \sum_{j=K_{1}}^{K_{2}-1}\left(c_{j+1, n}-c_{j n}\right)\left(M_{1}\left(X_{j+1: n}\right)-M_{1}\left(X_{j: n}\right)\right)- \\
& -n \sum_{j=K_{2}-1}^{n-1}\left(c_{j+1, n}-c_{j n}\right)\left(M_{2}\left(X_{j+2: n}\right)-M_{2}\left(X_{j+1: n}\right)\right)= \\
& =Z_{0}+Z_{1}+Z_{2} .
\end{aligned}
$$

First we estimate $\mathbb{E}|W|^{3}$. If $\alpha_{1} \geq \alpha_{2}$ then

$$
\left|c_{j n}\right| \leq a_{n} n^{-\left(\alpha_{1}-\alpha_{2}\right)},
$$

and using the estimation in Corollary 4.2 in Van Zwet (1984) we obtain

$$
\mathbb{E}|W|^{3} \leq C a_{n}^{3} n^{-3\left(\alpha_{1}-\alpha_{2}\right)} \mathbb{E}\left|X_{1}\right|^{3}
$$

Now let us consider the case $\alpha_{1}<\alpha_{2}$. First note that by Lemma 2

$$
\begin{aligned}
\mathbb{E} \int_{0}^{U_{1}} b_{j+1, n-j+1}(y) d F^{-1}(y)=\int_{0}^{1} \int_{0}^{x} b_{j+1, n-j+1}(y) d F^{-1}(y) d x= \\
\quad=\int_{0}^{1}(1-y) b_{j+1, n-j+1}(y) d F^{-1}(y) \\
\quad \leq K_{F} \frac{\Gamma(n+2)}{\Gamma(j+1) \Gamma(n-j+1)} \int_{0}^{1} y^{j-\kappa}(1-y)^{n-j+1-\kappa} d y \\
\quad=K_{F} \frac{\Gamma(n+2)}{\Gamma(j+1) \Gamma(n-j+1)} \frac{\Gamma(j+1-\kappa) \Gamma(n-j+2-\kappa)}{\Gamma(n+3-2 \kappa)} \\
\quad=K_{F} \mathcal{O}\left(n^{2 \kappa-1} j^{-\kappa}(n-j+1)^{1-\kappa}\right)=K_{F} \mathcal{O}\left(\left(\frac{j}{n}\right)^{-\kappa}\left(\frac{n-j+1}{n}\right)^{1-\kappa}\right) .
\end{aligned}
$$

In the same way for $j=1, \ldots, n-1$ we have

$$
\int_{0}^{1} b_{j+1, n-j+1}(y) d F^{-1}(y)=\mathcal{O}\left(\left(\frac{j}{n}\right)^{-\kappa}\left(\frac{n-j+1}{n}\right)^{-\kappa}\right),
$$

hence we obtain

$$
\begin{aligned}
& \mathbb{E}\left|H_{1 j}\left(U_{1}\right)\right|^{3}=\left(\frac{j}{n+1}\right)^{3} \mathbb{E}\left|\int_{0}^{U_{1}} b_{j+1, n-j+1}(y) d F^{-1}(y)\right|^{3} \leq \\
& \leq\left(\frac{j}{n+1}\right)^{3}\left(\int_{0}^{1} b_{j+1, n-j+1}(y) d F^{-1}(y)\right)^{2} \mathbb{E}\left|\int_{0}^{U_{1}} b_{j+1, n-j+1}(y) d F^{-1}(y)\right|= \\
& \quad=K_{F}^{3} \mathcal{O}\left(\left(\frac{j}{n}\right)^{3-3 \kappa}\left(\frac{n-j+1}{n}\right)^{1-3 \kappa}\right) .
\end{aligned}
$$

The statement above also holds for $j=n$, since the condition $\kappa<4 / 3$ implies

$$
\mathbb{E}\left|\int_{0}^{U_{1}} b_{j+1, n-j+1}(y) d F^{-1}(y)\right|^{3}=K_{F}^{3} \mathcal{O}\left(n^{3 \kappa-1}\right),
$$


as will be proved in the Appendix.

Similarly,

$$
\mathbb{E}\left|H_{2 j}\left(U_{1}\right)\right|^{3}=K_{F}^{3} \mathcal{O}\left(\left(\frac{j}{n}\right)^{1-3 \kappa}\left(\frac{n-j+1}{n}\right)^{3-3 \kappa}\right),
$$

hence

$$
\mathbb{E}\left|H_{1 j}\left(U_{1}\right)-H_{2 j}\left(U_{1}\right)\right|^{3}=K_{F}^{3} \mathcal{O}\left(\left(\frac{j}{n}\right)^{1-3 \kappa}\left(\frac{n-j+1}{n}\right)^{1-3 \kappa}\right) .
$$

Thus

$$
\begin{array}{r}
\mathbb{E}|W|^{3} \leq\left(a_{n} \sum_{j=1}^{n} n^{-1-\alpha_{1}}\left(\frac{j}{n}\right)^{-\alpha_{2}}\left(\frac{n-j+1}{n}\right)^{-\alpha_{2}} \sqrt[3]{\mathbb{E}\left|H_{1 j}\left(U_{1}\right)-H_{2 j}\left(U_{1}\right)\right|^{3}}\right)^{3}= \\
=a_{n}^{3} K_{F}^{3} \mathcal{O}\left(n^{-1-\alpha_{1}} \sum_{j=1}^{n}\left(\frac{j}{n}\right)^{1 / 3-\alpha_{2}-\kappa}\left(\frac{n-j+1}{n}\right)^{1 / 3-\alpha_{2}-\kappa}\right)^{3}=a_{n}^{3} K_{F}^{3} \mathcal{O}\left(\widetilde{A}_{n}\right) .
\end{array}
$$

Now we begin to analyse $\mathbb{E} Z^{2}$. If $\beta_{1} \geq \beta_{2}+1$ then

$$
\left|c_{j n}-c_{j-1, n}\right| \leq b_{n} n^{-\left(\beta_{1}-\beta_{2}\right)},
$$

and using the estimation in Corollary 4.2 in Van Zwet (1984) we obtain

$$
\mathbb{E} Z^{2} \leq C b_{n}^{2} n^{-2\left(\beta_{1}-\beta_{2}-1\right)} \mathbb{E} X_{1}^{2}
$$

Let us consider the case $\beta_{1}<\beta_{2}+1$. For $j=1, \ldots, n-1$ by Lemma 4

$$
\begin{aligned}
\mathbb{E}\left(\int_{U_{j-1: n}}^{U_{j: n}} x^{2} d F^{-1}(x)\right)^{2} & \leq K_{F}^{2} \mathbb{E}\left(\int_{U_{j-1: n}}^{U_{j: n}} x^{2-\kappa}(1-x)^{-\kappa} d x\right)^{2} \\
& =K_{F}^{2} \mathcal{O}\left(\frac{1}{n^{2}}\left(\frac{j}{n}\right)^{4-2 \kappa}\left(1-\frac{j}{n}\right)^{-2 \kappa}\right)
\end{aligned}
$$

hence

$$
\sqrt{\mathbb{E}\left(M_{0}\left(X_{j: n}\right)-M_{0}\left(X_{j-1: n}\right)\right)^{2}}=K_{F} \mathcal{O}\left(\frac{1}{n}\left(\frac{j}{n}\right)^{2-\kappa}\left(1-\frac{j}{n}\right)^{-\kappa}\right) .
$$

In a similar way we obtain for $1 \leq j \leq n-1$

$$
\begin{gathered}
\sqrt{\mathbb{E}\left(M_{1}\left(X_{j+1: n}\right)-M_{1}\left(X_{j: n}\right)\right)^{2}}=K_{F} \mathcal{O}\left(\frac{1}{n}\left(\frac{j}{n}\right)^{1-\kappa}\left(1-\frac{j}{n}\right)^{1-\kappa}\right), \\
\sqrt{\mathbb{E}\left(M_{2}\left(X_{j+2: n}\right)-M_{2}\left(X_{j+1: n}\right)\right)^{2}}=K_{F} \mathcal{O}\left(\frac{1}{n}\left(\frac{j}{n}\right)^{-\kappa}\left(1-\frac{j}{n}\right)^{2-\kappa}\right) .
\end{gathered}
$$


Berry-Esseen bound for L-statistics

First we estimate $\mathbb{E} Z_{1}^{2}$. Since the order statistics are independent of the ranks we have

$$
\begin{aligned}
\mathbb{E} Z_{1}^{2} & =\sum_{k_{1}=1}^{n-1} \sum_{k_{2}=k_{1}+1}^{n} \mathbb{E}\left\{Z_{1}^{2} \mid K_{1}=k_{1}, K_{2}=k_{2}\right\} \mathbb{P}\left\{K_{1}=k_{1}, K_{2}=k_{2}\right\}= \\
& =\frac{2 n}{n-1} \sum_{k_{1}=1}^{n-1} \sum_{k_{2}=k_{1}+1}^{n} \mathbb{E}\left(\sum_{j=k_{1}}^{k_{2}-1}\left(c_{j+1, n}-c_{j n}\right)\left(M_{1}\left(X_{j+1: n}\right)-M_{1}\left(X_{j: n}\right)\right)\right)^{2} .
\end{aligned}
$$

By (15)

$$
\begin{aligned}
& \sqrt{\mathbb{E}\left(\sum_{j=k_{1}}^{k_{2}-1}\left(c_{j+1, n}-c_{j n}\right)\left(M_{1}\left(X_{j+1: n}\right)-M_{1}\left(X_{j: n}\right)\right)\right)^{2}} \leq \\
& \leq b_{n} n^{-\beta_{1}} \sum_{j=k_{1}}^{k_{2}-1}\left(\frac{j}{n}\right)^{-\beta_{2}}\left(\frac{n-j+1}{n}\right)^{-\beta_{2}} \sqrt{\mathbb{E}\left(M_{1}\left(X_{j+1: n}\right)-M_{1}\left(X_{j: n}\right)\right)^{2}}= \\
& =K_{F} b_{n} n^{-\beta_{1}} \mathcal{O}\left(\frac{1}{n} \sum_{j=k_{1}}^{k_{2}-1}\left(\frac{j}{n}\right)^{1-\beta_{2}-\kappa}\left(1-\frac{j}{n}\right)^{1-\beta_{2}-\kappa}\right),
\end{aligned}
$$

thus

$$
\mathbb{E} Z_{1}^{2} \leq K_{F}^{2} b_{n}^{2} E_{1}
$$

where

$$
E_{1}=\mathcal{O}\left(\frac{1}{n^{2\left(1+\beta_{1}\right)}} \sum_{k_{1}=1}^{n-1} \sum_{k_{2}=k_{1}+1}^{n}\left(\sum_{j=k_{1}}^{k_{2}-1}\left(\frac{j}{n}\right)^{1-\beta_{2}-\kappa}\left(1-\frac{j}{n}\right)^{1-\beta_{2}-\kappa}\right)^{2}\right)
$$

We have

$$
n^{-1} \sum_{j=k_{1}}^{k_{2}-1}\left(\frac{j}{n}\right)^{1-\beta_{2}-\kappa}\left(1-\frac{j}{n}\right)^{1-\beta_{2}-\kappa}= \begin{cases}\mathcal{O}(1) & \text { if } \beta_{2}+\kappa<2, \\ \mathcal{O}\left(\log \frac{k_{2}}{n-k_{2}}-\log \frac{k_{1}}{n-k_{1}}\right) & \text { if } \beta_{2}+\kappa=2, \\ \mathcal{O}\left(\left(\frac{k_{1}}{n}\right)^{2-\beta_{2}-\kappa}+\left(1-\frac{k_{2}}{n}\right)^{2-\beta_{2}-\kappa}\right) & \text { if } \beta_{2}+\kappa>2 .\end{cases}
$$

In the case $\beta_{2}+\kappa<2$ we have

$$
E_{1}=\mathcal{O}\left(n^{-2 \beta_{1}} \sum_{k_{1}=1}^{n-1} \sum_{k_{2}=k_{1}+1}^{n} 1\right)=\mathcal{O}\left(n^{-2\left(\beta_{1}-1\right)}\right)
$$

In the case $\beta_{2}+\kappa=2$

$$
E_{1}=\mathcal{O}\left(n^{-2 \beta_{1}} \sum_{k_{1}=1}^{n-1} \sum_{k_{2}=k_{1}+1}^{n}\left(\log \frac{k_{2}}{n-k_{2}}+\log \frac{k_{1}}{n-k_{1}}\right)^{2}\right)=\mathcal{O}\left(n^{-2\left(\beta_{1}-1\right)}\right),
$$


since

$$
\int_{0}^{1} \int_{x_{1}}^{1}\left(\log \frac{x_{2}}{1-x_{2}}-\log \frac{x_{1}}{1-x_{1}}\right)^{2} d x_{2} d x_{1}<\infty .
$$

In the case $\beta_{2}+\kappa>2$

$$
\begin{aligned}
E_{1}= & \mathcal{O}\left(n^{-2 \beta_{1}} \sum_{k_{1}=1}^{n-1} \sum_{k_{2}=k_{1}+1}^{n}\left(\left(\frac{k_{1}}{n}\right)^{2-\beta_{2}-\kappa}+\left(1-\frac{k_{2}}{n}\right)^{2-\beta_{2}-\kappa}\right)^{2}\right) \\
= & \begin{array}{ll}
\mathcal{O}\left(n^{-2\left(\beta_{1}-1\right)}\right), & \text { if } 2<\beta_{2}+\kappa<\frac{5}{2} \\
\mathcal{O}\left(n^{-2\left(\beta_{1}-1\right)} \log n\right), & \text { if } \beta_{2}+\kappa=\frac{5}{2} \\
\mathcal{O}\left(n^{-2\left(\beta_{1}-\beta_{2}-\kappa\right)-3}\right), & \text { if } \beta_{2}+\kappa>\frac{5}{2}
\end{array}
\end{aligned}
$$

Now in a similar way we estimate $\mathbb{E} Z_{0}^{2}$. By (14)

$$
\begin{gathered}
\sqrt{\mathbb{E}\left(\sum_{j=1}^{k_{1}}\left(c_{j+1, n}-c_{j n}\right)\left(M_{0}\left(X_{j: n}\right)-M_{0}\left(X_{j-1: n}\right)\right)\right)^{2}}= \\
=K_{F} b_{n} n^{-\beta_{1}} \mathcal{O}\left(\frac{1}{n} \sum_{j=1}^{k_{1}}\left(\frac{j}{n}\right)^{2-\beta_{2}-\kappa}\left(1-\frac{j}{n}\right)^{-\beta_{2}-\kappa}\right),
\end{gathered}
$$

thus

$$
\mathbb{E} Z_{0}^{2} \leq K_{F}^{2} b_{n}^{2} E_{0}
$$

where

$$
E_{0}=\mathcal{O}\left(\frac{1}{n^{2\left(1+\beta_{1}\right)}} \sum_{k_{1}=1}^{n-1} \sum_{k_{2}=k_{1}+1}^{n}\left(\sum_{j=1}^{k_{1}}\left(\frac{j}{n}\right)^{2-\beta_{2}-\kappa}\left(1-\frac{j}{n}\right)^{-\beta_{2}-\kappa}\right)^{2}\right)
$$

We have

$$
\frac{1}{n} \sum_{j=1}^{k_{1}}\left(\frac{j}{n}\right)^{2-\beta_{2}-\kappa}\left(1-\frac{j}{n}\right)^{-\beta_{2}-\kappa}= \begin{cases}\mathcal{O}(1) & \text { if } \beta_{2}+\kappa<1, \\ \mathcal{O}\left(\log \frac{n}{n-k_{1}}\right) & \text { if } \beta_{2}+\kappa=1, \\ \mathcal{O}\left(\left(1-\frac{k_{1}}{n}\right)^{1-\beta_{2}-\kappa}\right) & \text { if } 1<\beta_{2}+\kappa<3, \\ \mathcal{O}\left(\log n+\left(1-\frac{k_{1}}{n}\right)^{-2}\right) & \text { if } \beta_{2}+\kappa=3, \\ \mathcal{O}\left(n^{\beta_{2}+\kappa-3}+\left(1-\frac{k_{1}}{n}\right)^{1-\beta_{2}-\kappa}\right) & \text { if } \beta_{2}+\kappa>3 .\end{cases}
$$

If $\beta_{2}+\kappa<1$ then

$$
E_{0}=\mathcal{O}\left(n^{-2 \beta_{1}} \sum_{k_{1}=1}^{n-1} \sum_{k_{2}=k_{1}+1}^{n} 1\right)=\mathcal{O}\left(n^{-2\left(\beta_{1}-1\right)}\right)
$$


If $\beta_{2}+\kappa=1$

$$
E_{0}=\mathcal{O}\left(n^{-2 \beta_{1}} \sum_{k_{1}=1}^{n-1} \sum_{k_{2}=k_{1}+1}^{n} \log ^{2}\left(1-\frac{k_{1}}{n}\right)\right)=\mathcal{O}\left(n^{-2\left(\beta_{1}-1\right)}\right)
$$

since

$$
\int_{0}^{1} \int_{x_{1}}^{1} \log ^{2}\left(1-x_{1}\right) d x_{2} d x_{1}<\infty .
$$

In the case $1<\beta_{2}+\kappa<3$

$$
E_{0}=\mathcal{O}\left(n^{-2 \beta_{1}} \sum_{k_{1}=1}^{n-1} \sum_{k_{2}=k_{1}+1}^{n}\left(1-\frac{k_{1}}{n}\right)^{2-2 \beta_{2}-2 \kappa}\right)=\mathcal{O}\left(n^{-2\left(\beta_{1}-1\right)}\right) .
$$

In the case $\beta_{2}+\kappa=3$

$$
E_{0}=\mathcal{O}\left(n^{-2 \beta_{1}} \sum_{k_{1}=1}^{n-1} \sum_{k_{2}=k_{1}+1}^{n}\left(\log ^{2} n+\left(1-\frac{k_{1}}{n}\right)^{-4}\right)\right)=\mathcal{O}\left(n^{-2\left(\beta_{1}-1\right)} \log ^{2} n\right) .
$$

In the case $\beta_{2}+\kappa>3$

$$
E_{0}=\mathcal{O}\left(n^{-2 \beta_{1}} \sum_{k_{1}=1}^{n-1} \sum_{k_{2}=k_{1}+1}^{n}\left(n^{2 \beta_{2}+2 \kappa-6}+\left(1-\frac{k_{1}}{n}\right)^{2-2 \beta_{2}-2 \kappa}\right)\right)=\mathcal{O}\left(n^{-2\left(\beta_{1}-\beta_{2}+2-\kappa\right)}\right) .
$$

Collecting the estimates we obtain

$$
E_{0}= \begin{cases}\mathcal{O}\left(n^{-2\left(\beta_{1}-1\right)}\right), & \text { if } \beta_{2}+\kappa<3 \\ \mathcal{O}\left(n^{-2\left(\beta_{1}-1\right)} \log ^{2} n\right), & \text { if } \beta_{2}+\kappa=3 \\ \mathcal{O}\left(n^{-2\left(\beta_{1}-\beta_{2}+2-\kappa\right)}\right), & \text { if } \beta_{2}+\kappa>3\end{cases}
$$

Because of symmetry the behaviour of $\mathbb{E} Z_{2}^{2}$ is the same. By

$$
\max \left\{E_{0}, E_{1}\right\}= \begin{cases}\mathcal{O}\left(n^{-2\left(\beta_{1}-1\right)}\right), & \text { if } \beta_{2}+\kappa<\frac{5}{2} \\ \mathcal{O}\left(n^{-2\left(\beta_{1}-1\right)} \log n\right), & \text { if } \beta_{2}+\kappa=\frac{5}{2} \\ \mathcal{O}\left(n^{-2\left(\beta_{1}-\beta_{2}-\kappa\right)-3}\right), & \text { if } \beta_{2}+\kappa>\frac{5}{2}\end{cases}
$$

the proof is complete.

\section{A law of large numbers for linear combinations of functions of order statistics}

In order to prove Theorem 2 and 3 we need a strong law of large numbers due to Van Zwet (1980) together with a corollary. 
Let $g:(0,1) \rightarrow \mathbb{R}$ be a Borel measurable function, and define $g_{n}:(0,1) \rightarrow \mathbb{R}$, $n=1,2, \ldots$, by

$$
g_{n}(t)=g\left(U_{[n t]+1: n}\right),
$$

where $[x]$ denotes the integer part of $x$. For $1<p<\infty, L_{p}$ is the Banach space of Lebesgue measurable functions $f:(0,1) \rightarrow \mathbb{R}$ with finite norm $\|f\|_{p}=\left(\int_{0}^{1}|f(t)|^{p} d t\right)^{1 / p}$. Let $K_{n} \in L_{p}, n=1,2, \ldots$ Consider

$$
\int_{0}^{1} K_{n}(t)\left(g_{n}(t)-g(t)\right) d t=\sum_{j=1}^{n} g\left(U_{j: n}\right) \int_{(j-1) / n}^{j / n} K_{n}(t) d t-\int_{0}^{1} K_{n}(t) g(t) d t .
$$

Recall a part of the statements of Theorem 2.1 and Corollary 2.1 in Van Zwet (1980):

Theorem A. Let $1<p<\infty, p^{-1}+q^{-1}=1$. If

(i) $\sup _{n}\left\|K_{n}\right\|_{p}<\infty$,

(ii) $\|g\|_{q}<\infty$,

then

$$
\lim _{n \rightarrow \infty} \int_{0}^{1} K_{n}(t)\left(g_{n}(t)-g(t)\right) d t=0 \quad \text { with probability } 1 .
$$

If, in addition, we assume that there exists a function $K \in L_{p}$ such that

(iii) $\lim _{n \rightarrow \infty} \int_{0}^{t} K_{n}(s) d s=\int_{0}^{t} K(s) d s$ for every $t \in(0,1)$,

then

$$
\lim _{n \rightarrow \infty} \int_{0}^{1} K_{n}(t) g_{n}(t) d t=\int_{0}^{1} K(t) g(t) d t \quad \text { with probability } 1 .
$$

One can deduce easily that the convergence $\int_{0}^{1} K_{n}(t) g_{n}(t) d t \rightarrow \int_{0}^{1} K(t) g(t) d t$ holds also in $L_{q}(\Omega, \mathcal{A}, \mathbb{P})$, where $(\Omega, \mathcal{A}, \mathbb{P})$ is the underlying probability space.

Lemma 5. Suppose that the conditions (i), (ii) and (iii) of Theorem A are satisfied. Then

$$
\lim _{n \rightarrow \infty} \mathbb{E}\left|\int_{0}^{1} K_{n}(t) g_{n}(t) d t-\int_{0}^{1} K(t) g(t) d t\right|^{q}=0 .
$$

Proof. Conditions (ii) and (iii) imply that $\int_{0}^{1} K_{n}(t) g(t) d t \rightarrow \int_{0}^{1} K(t) g(t) d t$, hence it is sufficient to prove that $\lim _{n \rightarrow \infty} \mathbb{E}\left|\int_{0}^{1} K_{n}(t)\left(g_{n}(t)-g(t)\right) d t\right|^{q}=0$. Hölder's inequality yields

$$
\left|\int_{0}^{1} K_{n}(t)\left(g_{n}(t)-g(t)\right) d t\right| \leq\left\|K_{n}\right\|_{p} \cdot\left\|g_{n}-g\right\|_{q},
$$


hence

$$
\mathbb{E}\left|\int_{0}^{1} K_{n}(t)\left(g_{n}(t)-g(t)\right) d t\right|^{q} \leq\left\|K_{n}\right\|_{p}^{q} \cdot \mathbb{E}\left\|g_{n}-g\right\|_{q}^{q}
$$

Obviously

$$
\mathbb{E}\left\|g_{n}\right\|_{q}^{q}=\mathbb{E} \int_{0}^{1}\left|g_{n}(t)\right|^{q} d t=n^{-1} \sum_{j=1}^{n} \mathbb{E}\left|g\left(U_{j}\right)\right|^{q}=\mathbb{E}\left|g\left(U_{1}\right)\right|^{q}=\int_{0}^{1}|g(t)|^{q} d t=\|g\|_{q}^{q} .
$$

As in the proof of Theorem 2.1 in Van Zwet (1980) we obtain that $\left\|g_{n}-g\right\|_{q} \rightarrow 0$ with probability 1 , thus by Vitali's theorem $\mathbb{E}\left\|g_{n}-g\right\|_{q}^{q} \rightarrow 0$.

\section{The proof of the remaining theorems}

Proof of Theorem 2. The proof will be given only in the case $\delta_{1}=1 / 2$. The case $\delta_{1}>1 / 2$ is simpler and can be treated in a similar way.

Let us define the step functions $\widetilde{J}_{n}:(0,1) \rightarrow \mathbb{R}$ and $\widetilde{\varphi}_{i n}:(0,1) \rightarrow \mathbb{R}, i=0,1, \ldots, k$, $n=1,2, \ldots$, for $(j-1) / n<t \leq j / n, j=1, \ldots, n$ by

$$
\widetilde{J}_{n}(t)=n \int_{(j-1) / n}^{j / n} J_{n}(t) d t=c_{j n}, \quad \widetilde{\varphi}_{i n}(t)=n \int_{(j-1) / n}^{j / n} \varphi_{i}(t) d t .
$$

We have

$$
\begin{aligned}
L_{n}= & n^{1 / 2} \int_{0}^{1} \widetilde{J}_{n}(t) \mathbb{F}_{n}^{-1}(t) d t= \\
= & n^{1 / 2} \int_{0}^{1} \widetilde{\varphi}_{0 n}(t) \mathbb{F}_{n}^{-1}(t) d t+\int_{0}^{1} \widetilde{\varphi}_{1 n}(t) \mathbb{F}_{n}^{-1}(t) d t+ \\
& +\mathcal{O}\left(n^{-1 / 2} \sum_{i=2}^{k} n^{-\delta_{i}} \sum_{j=1}^{n}\left(\frac{j}{n}\right)^{-\gamma_{i}}\left(\frac{n-j+1}{n}\right)^{-\gamma_{i}}\left|X_{j: n}\right|\right),
\end{aligned}
$$

where $\mathbb{F}_{n}$ denotes the empirical distribution function based on $X_{1}, \ldots, X_{n}$ :

$$
\mathbb{F}_{n}(t)=n^{-1} \sum_{j=1}^{n} 1_{(-\infty, t)}\left(X_{j}\right)
$$

and $\mathbb{F}_{n}^{-1}$ is the quantile function of $\mathbb{F}_{n}$. Using Hölder's inequality with $q=m$, $p^{-1}+q^{-1}=1$, and the law of large numbers we obtain for $i=2, \ldots, k$

$$
\begin{aligned}
& n^{-1 / 2-\delta_{i}} \sum_{j=1}^{n}\left(\frac{j}{n}\right)^{-\gamma_{i}}\left(\frac{n-j+1}{n}\right)^{-\gamma_{i}}\left|X_{j: n}\right| \\
& \quad \leq n^{-1 / 2-\delta_{i}}\left(\sum_{j=1}^{n}\left(\frac{j}{n}\right)^{-\gamma_{i} p}\left(\frac{n-j+1}{n}\right)^{-\gamma_{i} p}\right)^{1 / p}\left(\sum_{j=1}^{n}\left|X_{j}\right|^{q}\right)^{1 / q}=\mathcal{O}\left(\omega_{i n}\right) \quad \text { w.p.1, }
\end{aligned}
$$


where

$$
\omega_{i n}= \begin{cases}n^{\frac{1}{m}-\frac{1}{2}+\gamma_{i}-\delta_{i}} & \text { if } \gamma_{i} p>1 \\ n^{\frac{1}{2}-\delta_{i}}(\log n)^{\gamma_{i}} & \text { if } \gamma_{i} p=1 \\ n^{\frac{1}{2}-\delta_{i}} & \text { if } \gamma_{i} p<1\end{cases}
$$

Hence we conclude

$$
L_{n}=n^{1 / 2} \int_{0}^{1} \widetilde{\varphi}_{0 n}(t) \mathbb{F}_{n}^{-1}(t) d t+\int_{0}^{1} \widetilde{\varphi}_{1 n}(t) \mathbb{F}_{n}^{-1}(t) d t+\mathcal{O}\left(\sum_{i=2}^{k} \omega_{i n}\right) \quad \text { w.p.1. }
$$

We remark that the conditions (i) and (iv) implies $\omega_{\text {in }}=o(1)$ for $i=2, \ldots, k$.

Now we apply Theorem A and Lemma 5 with $g=F^{-1}, \quad q=m, K=\varphi_{1}$ and $K_{n}=\widetilde{\varphi}_{1 n}$. First we check the conditions. Straightforward calculation leads for some $c>0$ to

$$
\left|K_{n}(t)\right| \leq c\left\|\psi_{1}\right\|_{\infty}\left(\frac{j}{n}\right)^{-\gamma_{1}}\left(\frac{n-j+1}{n}\right)^{-\gamma_{1}} \quad \text { for } \quad \frac{j-1}{n}<t \leq \frac{j}{n}, j=1, \ldots, n,
$$

which implies for some $\widetilde{c}>0$

$$
\left\|K_{n}\right\|_{p}^{p} \leq \widetilde{c}\left\|\psi_{1}\right\|_{\infty}^{p} \int_{0}^{1} t^{-\gamma_{1} p}(1-t)^{-\gamma_{1} p} d t<\infty
$$

since $q=m>\left(1-\gamma_{1}\right)^{-1}$ implies $\gamma_{1} p<1$. Moreover, $\|g\|_{q}^{q}=\mathbb{E}\left|X_{1}\right|^{q}=\mathbb{E}\left|X_{1}\right|^{m}<\infty$. By the help of the equality

$$
\int_{(j-1) / n}^{j / n} \widetilde{\varphi}_{1 n}(u) d u=\int_{(j-1) / n}^{j / n} \varphi_{1}(u) d u
$$

we have for $(j-1) / n<t \leq j / n$

$$
\left|\int_{0}^{t} K_{n}(u) d u-\int_{0}^{t} K(u) d u\right| \leq \int_{(j-1) / n}^{j / n}\left|\varphi_{1}(u)\right| d u=\left\|\psi_{1}\right\|_{\infty} \mathcal{O}\left(n^{-1} t^{-\gamma_{1}}(1-t)^{-\gamma_{1}}\right),
$$

which implies that condition (iii) holds. Hence we can use Theorem A and Lemma 5 and obtain

$$
\lim _{n \rightarrow \infty} \int_{0}^{1} \widetilde{\varphi}_{1 n}(t) \mathbb{F}_{n}^{-1}(t) d t=\mu\left(\varphi_{1}, F\right) \quad \text { w.p.1 }
$$

and

$$
\lim _{n \rightarrow \infty} \mathbb{E}\left|\int_{0}^{1} \widetilde{\varphi}_{1 n}(t) \mathbb{F}_{n}^{-1}(t) d t-\mu\left(\varphi_{1}, F\right)\right|^{m}=0
$$

where

$$
\mu\left(\varphi_{i}, F\right)=\int_{0}^{1} \varphi_{i}(t) F^{-1}(t) d t
$$

Combining (18) and (19) we get

$$
L_{n}=n^{1 / 2} \int_{0}^{1} \widetilde{\varphi}_{0 n}(t) \mathbb{F}_{n}^{-1}(t) d t+\mu\left(\varphi_{1}, F\right)+o(1) \quad \text { w.p.1. }
$$


The conditions $0 \leq \gamma_{0}<1 / 2$ and $m>\left(1 / 2-\gamma_{0}\right)^{-1}$ imply $m>2$, hence by (20) we obtain

$$
\lim _{n \rightarrow \infty} \mathbb{E} \int_{0}^{1} \widetilde{\varphi}_{1 n}(t) \mathbb{F}_{n}^{-1}(t) d t=\mu\left(\varphi_{1}, F\right)
$$

and (20) together with (22) imply

$$
\lim _{n \rightarrow \infty} \sigma^{2}\left(\int_{0}^{1} \widetilde{\varphi}_{1 n}(t) \mathbb{F}_{n}^{-1}(t) d t\right)=0
$$

Using Theorem 1 in Mason (1981) with $r=s=\left(1 / 2-\gamma_{0}\right)^{-1}$ and the weight function $J=\varphi_{0}$ we obtain for the linear combinations

$$
S_{n}=n^{-1 / 2} \sum_{j=1}^{n} \varphi_{0}\left(\frac{j}{n+1}\right) X_{j: n}
$$

the CLT

$$
S_{n}-\mathbb{E} S_{n} \stackrel{\mathcal{D}}{\longrightarrow} \mathcal{N}\left(0, \sigma^{2}\left(\varphi_{0}, F\right)\right)
$$

and

$$
\lim _{n \rightarrow \infty} \sigma^{2}\left(S_{n}\right)=\sigma^{2}\left(\varphi_{0}, F\right)
$$

(We remark that the condition $\mathbb{E}\left|X_{1}\right|^{m}<\infty$ for some $m>\left(\frac{1}{2}-\gamma_{0}\right)^{-1}$ implies that Mason's condition

$$
\int_{0}^{1} x^{\frac{1}{2}-\gamma_{0}}(1-x)^{\frac{1}{2}-\gamma_{0}} d F^{-1}(x)<\infty
$$

holds.)

Let

$$
T_{n}=n^{1 / 2} \int_{0}^{1} \widetilde{\varphi}_{0 n}(t) \mathbb{F}_{n}^{-1}(t) d t
$$

To prove

$$
T_{n}-\mathbb{E} T_{n} \stackrel{\mathcal{D}}{\longrightarrow} \mathcal{N}\left(0, \sigma^{2}\left(\varphi_{0}, F\right)\right)
$$

and

$$
\lim _{n \rightarrow \infty} \sigma^{2}\left(T_{n}\right)=\sigma^{2}\left(\varphi_{0}, F\right)
$$

it is sufficient to show

$$
\limsup _{n \rightarrow \infty} \sigma^{2}\left(S_{n}-T_{n}\right)=0 .
$$

We have

$$
S_{n}-T_{n}=n^{1 / 2} \sum_{j=1}^{n} d_{j n} X_{j: n},
$$


where

$$
d_{j n}=\int_{(j-1) / n}^{j / n}\left(\varphi_{0}\left(\frac{j}{n+1}\right)-\varphi_{0}(t)\right) d t
$$

By Hölder's inequality with $q=m, p^{-1}+q^{-1}=1$

$$
\begin{aligned}
& \sigma^{2}\left(\sum_{j=1}^{n} d_{j n} X_{j: n}\right)=\mathbb{E}\left(\sum_{j=1}^{n} d_{j n}\left(X_{j: n}-\mathbb{E} X_{j: n}\right)\right)^{2} \leq \\
& \leq\left(\sum_{j=1}^{n}\left|d_{j n}\right|^{p}\right)^{2 / p} \mathbb{E}\left(\sum_{j=1}^{n}\left|X_{j: n}-\mathbb{E} X_{j: n}\right|^{q}\right)^{2 / q}=\mathcal{O}\left(n^{2 / q}\left(\sum_{j=1}^{n}\left|d_{j n}\right|^{p}\right)^{2 / p}\right) .
\end{aligned}
$$

By

$$
\begin{aligned}
\left|d_{j n}\right| \leq & \Lambda_{0}\left(\frac{j}{n+1}\right)^{-\gamma_{0}}\left(\frac{n-j+1}{n+1}\right)^{-\gamma_{0}} \int_{(j-1) / n}^{j / n}\left|\frac{j}{n+1}-t\right|^{\lambda_{0}} d t+ \\
& +\gamma_{0}\left\|\psi_{0}\right\|_{\infty}\left(\frac{j}{n+1}\right)^{-\gamma_{0}-1}\left(\frac{n-j+1}{n+1}\right)^{-\gamma_{0}-1} \int_{(j-1) / n}^{j / n}\left|\frac{j}{n+1}-t\right| d t= \\
\leq & \Lambda_{0} n^{-\lambda_{0}-1}\left(\frac{j}{n+1}\right)^{-\gamma_{0}}\left(\frac{n-j+1}{n+1}\right)^{-\gamma_{0}}+ \\
& +\gamma_{0}\left\|\psi_{0}\right\|_{\infty} n^{-2}\left(\frac{j}{n+1}\right)^{-\gamma_{0}-1}\left(\frac{n-j+1}{n+1}\right)^{-\gamma_{0}-1}
\end{aligned}
$$

we obtain

$$
\left(\sum_{j=1}^{n}\left|d_{j n}\right|^{p}\right)^{2 / p} \leq n^{-2 \lambda_{0}-2} A^{2 / p}+n^{-4} B^{2 / p}
$$

where

$$
\begin{gathered}
A=\mathcal{O}\left(\sum_{j=1}^{n}\left(\frac{j}{n+1}\right)^{-\gamma_{0} p}\left(\frac{n-j+1}{n+1}\right)^{-\gamma_{0} p}\right)=\mathcal{O}(n), \\
B=\mathcal{O}\left(\sum_{j=1}^{n}\left(\frac{j}{n+1}\right)^{-\left(\gamma_{0}+1\right) p}\left(\frac{n-j+1}{n+1}\right)^{-\left(\gamma_{0}+1\right) p}\right)=\mathcal{O}\left(n^{\left(\gamma_{0}+1\right) p}\right) .
\end{gathered}
$$

Collecting the estimates we conclude

$$
\sigma^{2}\left(S_{n}-T_{n}\right)=\mathcal{O}\left(n^{1+2 / q}\left(n^{-2 \lambda_{0}-2+2 / p}+n^{2 \gamma_{0}-2}\right)\right)=\mathcal{O}\left(n^{1-2 \lambda_{0}}+n^{2 / q+2 \gamma_{0}-1}\right)=o(1),
$$

hence we verified (26). By (25) and (23) we obtain

$$
\lim _{n \rightarrow \infty} \sigma^{2}\left(n^{1 / 2} \int_{0}^{1} \widetilde{\varphi}_{0 n}(t) \mathbb{F}_{n}^{-1}(t) d t+\int_{0}^{1} \widetilde{\varphi}_{1 n}(t) \mathbb{F}_{n}^{-1}(t) d t\right)=\sigma^{2}\left(\varphi_{0}, F\right),
$$

which together with (18) implies

$$
\lim _{n \rightarrow \infty} \sigma^{2}\left(L_{n}\right)=\sigma^{2}\left(\varphi_{0}, F\right) .
$$


Now (21) and (24) yields

$$
L_{n}-\mathbb{E} L_{n} \stackrel{\mathcal{D}}{\longrightarrow} \mathcal{N}\left(0, \sigma^{2}\left(\varphi_{0}, F\right)\right)
$$

Hence the proof is completed for $L_{n}$.

Now let us define the step functions $\widehat{J}_{n}:(0,1) \rightarrow \mathbb{R}$ and $\widehat{\varphi}_{i n}:(0,1) \rightarrow \mathbb{R}, \quad i=$ $0,1, \ldots, k, n=1,2, \ldots$, for $(j-1) / n<t \leq j / n, j=1, \ldots, n$ by

$$
\widehat{J}_{n}(t)=J_{n}\left(\frac{j}{n+1}\right)=\widehat{c}_{j n}, \quad \widehat{\varphi}_{i n}(t)=\varphi_{n}\left(\frac{j}{n+1}\right) .
$$

As earlier we can come to the conclusion

$$
\widehat{L}_{n}=n^{1 / 2} \int_{0}^{1} \widehat{\varphi}_{0 n}(t) \mathbb{F}_{n}^{-1}(t) d t+\int_{0}^{1} \widehat{\varphi}_{1 n}(t) \mathbb{F}_{n}^{-1}(t) d t+\mathcal{O}\left(\sum_{i=2}^{k} \omega_{i n}\right) \quad \text { w.p.1. }
$$

We apply Theorem A and Lemma 5 with $g=F^{-1}, q=m, K=\varphi_{1}$ and $K_{n}=\widehat{\varphi}_{1 n}$. The conditions (i) and (ii) can be checked as before. Using the earlier arguments we obtain for $j=1, \ldots, n$

$$
\begin{aligned}
& \int_{(j-1) / n}^{j / n}\left(\varphi_{1}\left(\frac{j}{n+1}\right)-\varphi_{1}(t)\right) d t \leq \\
& \leq \Lambda_{1} n^{-\lambda_{1}-1}\left(\frac{j}{n+1}\right)^{-\gamma_{1}}\left(\frac{n-j+1}{n+1}\right)^{-\gamma_{1}}+ \\
& \quad+\gamma_{1}\left\|\psi_{1}\right\|_{\infty} n^{-2}\left(\frac{j}{n+1}\right)^{-\gamma_{1}-1}\left(\frac{n-j+1}{n+1}\right)^{-\gamma_{1}-1}
\end{aligned}
$$

We have for $(j-1) / n<t \leq j / n$

$$
\left|\int_{0}^{t} K_{n}(u) d u-\int_{0}^{t} K(u) d u\right| \leq \sum_{j=1}^{n} \int_{(j-1) / n}^{j / n}\left|\varphi_{1}\left(\frac{j}{n+1}\right)-\varphi_{1}(u)\right| d u=\mathcal{O}\left(n^{-\lambda_{1}}+n^{\gamma_{1}-1}\right)
$$

which implies that condition (iii) holds. Applying Theorem A, Lemma 5, and then Theorem 1 in Mason (1981) again, completes the proof.

Proof of Theorem 3. The coefficient $c_{j n}$ can be decomposed as follows.

$$
c_{j n}=\sum_{i=0}^{k} c_{j n}^{(i)}, \quad \text { where } \quad c_{j n}^{(i)}=n^{1-\delta_{i}} \int_{(j-1) / n}^{j / n} \varphi_{i}(t) d t
$$

Clearly

$$
\left|c_{j n}^{(i)}\right|=\frac{\left\|\psi_{i}\right\|_{\infty}}{1-\gamma_{i}} \mathcal{O}\left(n^{-\delta_{i}}\left(\frac{j}{n}\right)^{-\gamma_{i}}\left(\frac{n-j+1}{n}\right)^{-\gamma_{i}}\right)
$$




$$
\begin{aligned}
\left|c_{j n}^{(i)}-c_{j-1, n}^{(i)}\right|=\mathcal{O}( & \Lambda_{i} n^{-\delta_{i}-\lambda_{i}}\left(\frac{j}{n}\right)^{-\gamma_{i}}\left(\frac{n-j+1}{n}\right)^{-\gamma_{i}}+ \\
& \left.+\frac{\left\|\psi_{i}\right\|_{\infty}}{1-\gamma_{i}} n^{-\delta_{i}-1}\left(\frac{j}{n}\right)^{-\gamma_{i}-1}\left(\frac{n-j+1}{n}\right)^{-\gamma_{i}-1}\right) .
\end{aligned}
$$

Applying the inequality (12) and using the estimation method as given in the proof of Theorem 1 for each index $i=0,1, \ldots, k$ the proof can be completed.

\section{Appendix}

Proof of Lemma 1. $p_{0 n}$ is the unique solution of $(1-p)^{n}=\alpha$, hence $p_{0 n}=1-\sqrt[n]{\alpha}$, which implies

$$
r_{0}:=\lim _{n \rightarrow \infty} n p_{0 n}=\log \frac{1}{\alpha}
$$

thus $r_{0}$ is the solution of

$$
\alpha e^{r_{0}}=1
$$

For $n \geq 2, p_{1 n}$ is the unique solution of $n p(1-p)^{n-1}+(1-p)^{n}=\alpha$, hence $p_{1 n}=h\left(n^{-1}\right)$, where $h:(0,1) \rightarrow(0,1)$ is the uniquely defined function such that

$$
\frac{1}{u} h(u)(1-h(u))^{1 / u-1}+(1-h(u))^{1 / u}=\alpha .
$$

Obviously $\lim _{u \rightarrow 0} h(u)=\lim _{n \rightarrow \infty} p_{1 n}=0$, hence by $h(0):=0$ the definition of the function $h(u)$ can be extended to $u=0$ by continuity. Moreover, the function $h$ is differentiable.

Clearly $x_{n}:=n p_{1 n}=n h\left(n^{-1}\right)$ is the unique solution of

$$
x_{n}\left(1-\frac{x_{n}}{n}\right)^{n-1}+\left(1-\frac{x_{n}}{n}\right)^{n}=\alpha .
$$

Consequently,

$$
r_{1}:=h^{\prime}(0)=\lim _{n \rightarrow \infty} n h\left(n^{-1}\right)=\lim _{n \rightarrow \infty} x_{n}=\lim _{n \rightarrow \infty} n p_{1 n}
$$

is the unique positive solution of

$$
\alpha e^{r_{1}}=1+r_{1}
$$

Similarly, $r_{2}:=\lim _{n \rightarrow \infty} n p_{2 n}$ is the unique positive solution of

$$
\alpha e^{r_{2}}=1+r_{2}+\frac{1}{2} r_{2}^{2}
$$

We can conclude

$$
\lim _{n \rightarrow \infty} n\left(p_{2, n}-2 p_{1, n}+p_{0 n}\right)=r_{2}-2 r_{1}+r_{0} \neq 0
$$


Berry-Esseen bound for L-statistics

hence the assertion holds.

Proof of (13). First we note that

$$
\begin{aligned}
\mathbb{E}\left|\int_{0}^{U_{1}} b_{j+1, n-j+1}(y) d F^{-1}(y)\right|^{3} & =\int_{0}^{1}\left(\int_{0}^{x}(n+1) y^{n} d F^{-1}(y)\right)^{3} d x \\
& \leq c K_{F}^{3} n^{3} \int_{0}^{1}\left(\int_{0}^{x} y^{n-\kappa}(1-y)^{-\kappa} d y\right)^{3} d x
\end{aligned}
$$

hence we have to show

$$
\int_{0}^{1}\left(\int_{0}^{x} y^{n-\kappa}(1-y)^{-\kappa} d y\right)^{3} d x=\mathcal{O}\left(n^{3 \kappa-4}\right) .
$$

If $1<\kappa<4 / 3$ then

$$
\int_{0}^{x} y^{n-\kappa}(1-y)^{-\kappa} d y \leq x^{n-\kappa} \int_{0}^{x}(1-y)^{-\kappa} d y<\frac{x^{n-\kappa}(1-x)^{1-\kappa}}{\kappa-1}
$$

and by Lemma 1 we have

$$
\int_{0}^{1} x^{3(n-\kappa)}(1-x)^{3(1-\kappa)} d x=\frac{\Gamma(3 n-3 \kappa+1) \Gamma(4-3 \kappa)}{\Gamma(3 n-6 \kappa+5)}=\mathcal{O}\left(n^{3 \kappa-4}\right),
$$

hence we obtain (27).

If $0 \leq \kappa<1$ then

$$
\int_{0}^{1} \int_{0}^{x} y^{n-\kappa}(1-y)^{-\kappa} d y d x=\int_{0}^{1} y^{n-\kappa}(1-y)^{1-\kappa} d y=\frac{\Gamma(n-\kappa+1) \Gamma(2-\kappa)}{\Gamma(n-2 \kappa+3)}=\mathcal{O}\left(n^{\kappa-2}\right)
$$

and

$$
\int_{0}^{1} y^{n-\kappa}(1-y)^{-\kappa} d y=\frac{\Gamma(n-\kappa+1) \Gamma(1-\kappa)}{\Gamma(n-2 \kappa+2)}=\mathcal{O}\left(n^{\kappa-1}\right)
$$

hence

$$
\begin{aligned}
\int_{0}^{1}\left(\int_{0}^{x} y^{n-\kappa}(1-y)^{-\kappa} d y\right)^{3} d x & \leq\left(\int_{0}^{1} y^{n-\kappa}(1-y)^{-\kappa} d y\right)^{2} \int_{0}^{1} \int_{0}^{x} y^{n-\kappa}(1-y)^{-\kappa} d y d x \\
& =\mathcal{O}\left(n^{2(\kappa-1)} n^{\kappa-2)}\right)=\mathcal{O}\left(n^{3 \kappa-4}\right) .
\end{aligned}
$$

In the case $\kappa=1$ the estimation (27) can be proved, for example, by Taylor expansion of the integrand.

Acknowledgement. The authors are indebted to W.R. van Zwet for careful reading an earlier version of this paper and for valuable comments. 


\section{References}

Anderson, R. J. and A. D. Teitlebaum (1973), Dollar-unit sampling, Canad. Chartered Accountant (after 1973, this publication became CA Magazine) April, 30-39.

Bentkus, V., F. Götze and A. Tikhomirov, (1997). Berry-Esseen bound for statistics of weakly dependent samples, Bernoulli 3, 329-350.

Bentkus, V., F. Götze and W. R. van Zwet, (1994). An Edgeworth expansion for symmetric 0, Preprint No. 94-020 Sonderforschungsbereich 343, Diskrete Strukturen in der Mathematik, Bielefeld.

Bickel, P. J. (1992), Inference and auditing: the Stringer bound, International Statistical Review 60, 197-209.

BJERve, S. (1977), Error bounds for linear combinations of order statistics, Ann. Statist. 5, $357-369$.

David, H. A. (1981), Order Statistics, John Wiley \& Sons, New York.

FriedrICH, K. O. (1985), Berry-Esseen Abschätzungen für Nichtparametrische Statistiken, Dissertation, Albert-Ludwig-Universität, Freiburg in Breisgau.

Friedrich, K. O. (1989), A Berry-Esseen bound for independent random variables, Ann. Statist. 17, 170-184.

Helmers, R. (1981), A Berry-Esseen theorem for linear combinations of order statistics, The Ann. Probab. 9, 342-347.

Helmers, R. (1982), Edgeworth expansions for linear combinations of order statistics, Amsterdam, Math. Centre Tracts 105.

Helmers, R. and M. Hušková (1984), A Berry-Esseen bound for L-statistics with unbounded weight functions, Proc. Third Prague Symp. on Asymptotic Statistics, 93-101.

Helmers, R. and M. HušKová (1986), Berry-Esseen bounds for $L$-statistics with unbounded weight functions, Report MS-R8611, Centre for Mathematics and Computer Science, Amsterdam.

De Jager, N. G., G. Pap and M. C. A. van Zuijlen (1997), Facts, phantasies and a new proposal concerning the Stringer bound, Computers Math. Applic. 33, 37-54.

Leslie, D. A., A. D. Teitlebaum and R. J. Anderson (1980), Dollar-Unit Sampling - A Practical Guide for Auditors. Pitman, London.

Mason, M. M. (1981), Asymptotic normality of linear combinations of order statistics with a smooth score function, Ann. Statist. 9, 899-908.

PAP, G. and M. C. A. van ZuiJlen (1994), On the Stringer bound in case of uniform taintings, Computers Math. Applic. 29, 51-59.

Pap, G. and M. C. A. van ZuiJlen (1996), On the asymptotic behaviour of the Stringer bound, Statistica Neerlandica 50, 367-389.

PArzen, E. (1979), Nonparametric statistical data modelling, JASA 74, 105-121. 
REISs, R.-D. (1989), Approximate distributions of order statistics, Springer-Verlag, New York Berlin Heidelberg.

Shorack, G. R. and J. A. Wellner (1986), Empirical processes with applications to statistics. John Wiley \& Sons, New York.

Schuster, E. F. (1984), Classification of probability laws by tail behavior, JASA 79, 936-939.

Serfling, R. J. (1980), Approximation Theorems of Mathematical Statistics, John Wiley \& Sons, New York.

Statistical Models and Analysis in Auditing (1989). Statistical Science 4, 2-33.

VAn Zwet, W. R. (1980), A strong law for linear functions of order statistics, The Ann. Probab. 8, 986-990.

VAn Zwet, W. R. (1984), A Berry-Esseen bound for symmetric statistics, Z. Wahrsch. verw. Gebiete 66, 425-440. 